


\section{DISCLAIMER}

This report was prepared as an account of work sponsored by an agency of the United States Government. Neither the United States Government nor any agency Thereof, nor any of their employees, makes any warranty, express or implied, or assumes any legal liability or responsibility for the accuracy, completeness, or usefulness of any information, apparatus, product, or process disclosed, or represents that its use would not infringe privately owned rights. Reference herein to any specific commercial product, process, or service by trade name, trademark, manufacturer, or otherwise does not necessarily constitute or imply its endorsement, recommendation, or favoring by the United States Government or any agency thereof. The views and opinions of authors expressed herein do not necessarily state or reflect those of the United States Government or any agency thereof. 


\section{DISCLAIMER}

Portions of this document may be illegible in electronic image products. Images are produced from the best available original document. 
Printed in the United States of America. Available from National Technical Information Service

U. S. Department of Commerce

5285 Port Royal Road

Springfield, Virginia 22151

Price: Printed Copy \$300; Microfiche \$0.95

$-4,100$ 


\title{
Petrography of Some Rock Types of the Precambrian Basement Near the Los Alamos Scientific Laboratory Geothermal Test Site, Jemez Mountains, New Mexico
}

by

\author{
Priscilla C. Perkins*
}

*Institute of Geophysics and Planetary Physics, University of California, Los Angeles. 


\title{
PETROGRAPHY OF SOME ROCK TYPES OF THE PRECAMBRIAN BASEMENT
}

\begin{abstract}
NEAR THE LOS ALAMOS SCIENTIFIC LABORATORY GEOTHERMAL TEST SITE,
\end{abstract}
JEMEZ MOUNTAINS, NEW MEXICO

by

Priscilla C. Perkins

\begin{abstract}
The proposed geothermal energy experiment by the Los Alamos Scientific Laboratory is to be conducted in Precambrian rocks underlying the Jeme\% Platcau, New Mexico. This study describes 25 samples from $152 \mathrm{ft}$ of drill core taken from Granite Test Hole One, and 12 examples of Precambrian rocks of the region not far distant from the proposed geothermal test site.

The upper $94 \mathrm{ft}$ of Precambrian rock in the test hole is granite and granodiorite, partly gneissic in texture. The lower $61 \mathrm{ft}$ is biotite-amphibolite veined by tonalite-aplite.

All cracks observed in thin section follow thin calcite veins. In hand specimen, chlorite-lined fracture surfaces are also present.

Rocks of the drill corc provide a good variety of compositions representative of many of the rock types collected for this study from regional localities (Sandia Mountains, San Pedro Mountain, Soda Dam, Guadalupe Box, and the Nacimiento Mountains). As fresh samples, they will provide suitable material for hydrothermal experiments.

The presence of "red granite" xenoliths in pyroclastic rocks of the Jemez Plateau, and the predominance of granitoid rocks over amphibolite in regional outcrops suggest that granitic rocks are the predominant rock type below the test site.
\end{abstract}

\section{INTRODUCTION}

The proposed geothermal energy experiment by the Los Alamos Scientific Laboratory is to be conducted in Precambrian rocks underlying Paleozoic and Mesozoic sediments and Cenozoic volcanics of the Jemez Plateau, New Mexico. In the experiment, water will be circulated at depth in the Precambrian basement.

The purpose of this petrographic study is to provide information pertinent to the following questions:

1. What are the likely host rocks for the experiment, which is to be carried out at depth below the level of present drill holes?
2. What will be the chemical effects of circulating hot fluids in fractured basement rocks?

Until further test holes are drilled, the first question can be addressed only indirectly. The petrographic study secks to establish the range of typical rock types present in recovered drill core and in available regional Precambrian exposures in the general area. If areal variation in the Precambrian terrain exposed at the surface is similar to variability with depth in the basement, a general range of likely host rock types can be established. Toward this end, this report characterizes samples from two sources: 1) drill core from Granite Test Hole One, Barley 
Canyon, Jemez Plateau; and 2) outcrops of Precambrian rocks selected because of their proximity to the proposed geothermal test sitc.

After anticipated host rock types have been identified, laboratory experiments can be begun to establish the nature of chemical reactions between hot fluids and the rocks at the temperatures expected in the geothermal experiment. The petrographic study provides baseline information on the samples for experimental study by tabulating identity and abundance of mineral constituents, mincralogy of vein and fracture fillings, and frequency and identity of crystal-crystal contacts in the rock matrix along which fluids may be expected to pass.

\section{LOCATION OF SAMPLES}

Granite Test Hole One, near the proposed test site, was drilled during May 1972 in Barley Canyon, Jemez Mountains. An interval of $155 \mathrm{ft}$ in Precambrian granite and amphibolite was penetrated. Table I lists the locations of samples from the recovered drill core sectioned for petrographic examination.

Precambrian outcrops near the drill site were sought, but unfortunately the closest exposures available were 13 to 16 miles distant.' ligure 1 shows the locations sampled. Localities are described in Table II.

TABLE I

\section{LOCATION OF SAMPLES FROM GRANITE TEST HOLE ONE}

(Samples numbered and located by R. Potter)

\begin{tabular}{l} 
Thin Section \\
Sample Number \\
\hline PD-2-a,b,c \\
PD-2-1 \\
PD-3-5 \\
PD-2-2 \\
PD-2-3 \\
PD-2-4 \\
PD-2-5 \\
PD-3-7 \\
PD-2-6 \\
PD-2-7 \\
PD-2-8 \\
PD-2-12 \\
PD-3-6 \\
PD-2-13 \\
PD-2-14 \\
PD-3-8 \\
PD-2-15 \\
PD-2-9 \\
PD-2-11 \\
PD-2-10 \\
PD-2-16 \\
PD-3-1 \\
PD-3-3 \\
PD-3-2 \\
PD-3-4
\end{tabular}

End of core

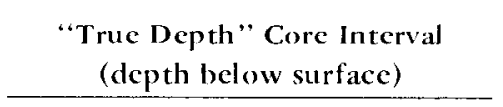

$2422 \mathrm{ft}$ (approximate)

$2329 \mathrm{ft}, 5 \mathrm{in}$ to $2329 \mathrm{ft}, 8-1 / 2 \mathrm{in}$.

$2437 \mathrm{ft}, 11 \mathrm{in.}$ to $2438 \mathrm{ft}, 1-1 / 2 \mathrm{in}$.

$2444 \mathrm{ft}$ to $2444 \mathrm{ft}, 4 \mathrm{in}$.

$2466 \mathrm{ft}, 4 \mathrm{in}$. to $2467 \mathrm{ft}$

$2481 \mathrm{ft}$ to $2481 \mathrm{ft}, 3 \mathrm{in}$.

$2484 \mathrm{ft}, 2$ in. to $2484 \mathrm{ft}, 4 \mathrm{in}$.

$2497 \mathrm{ft}$ (approximate)

$2500 \mathrm{ft}, 7 \mathrm{in} . \mathrm{to} 2500 \mathrm{ft}, 9-1 / 2 \mathrm{in}$.

$2501 \mathrm{ft}, 8 \mathrm{in}, \mathrm{to} 2502 \mathrm{ft}$

$2510 \mathrm{ft}, 3 \mathrm{in}$. to $2510 \mathrm{ft}, 6 \mathrm{in}$.

$2515 \mathrm{ft}, 6 \mathrm{in}$. (approximate)

$2516 \mathrm{ft}, 3-1 / 2 \mathrm{in}$. to $2516 \mathrm{ft}, 5-1 / 2 \mathrm{in}$.

$2517 \mathrm{ft}, 9$ in. to $2518 \mathrm{ft}$

$2519 \mathrm{ft}, 3 \mathrm{in}$. to $2519 \mathrm{ft}, 8 \mathrm{in}$.

$2520 \mathrm{ft}$ (approximate)

$2520 \mathrm{ft}, 10-1 / 2 \mathrm{in}$. to $2521 \mathrm{ft}, 8 \mathrm{in}$.

$2527 \mathrm{ft}, 1 \mathrm{in}$ to $2527 \mathrm{ft}, 3-1 / 2 \mathrm{in}$.

$2529 \mathrm{ft}, 6 \mathrm{in} . \mathrm{to} 2529 \mathrm{ft}, 10 \mathrm{in}$.

$2530 \mathrm{ft}, 2-1 / 2 \mathrm{in}$. to $2530 \mathrm{ft}, 5-1 / 2 \mathrm{in}$.

$2541 \mathrm{ft}, 9 \mathrm{in}$. to $2542 \mathrm{ft}$

$2548 \mathrm{ft}, 10-1 / 2 \mathrm{in}$. to $2549 \mathrm{ft}, 1 \mathrm{in}$.

$2556 \mathrm{ft}, 11 \mathrm{in}$. to $2557 \mathrm{ft}, 2-1 / 2 \mathrm{in}$.

$2559 \mathrm{ft}, 3-1 / 2$ in. to $2559 \mathrm{ft}, 5 \mathrm{in}$.

$2573 \mathrm{ft}, 11-1 / 2 \mathrm{in}$. to $2574 \mathrm{ft}, 3 \mathrm{in}$.

\author{
Cumulative Depth \\ below 2422-ft Level \\ (nearest foot)
}




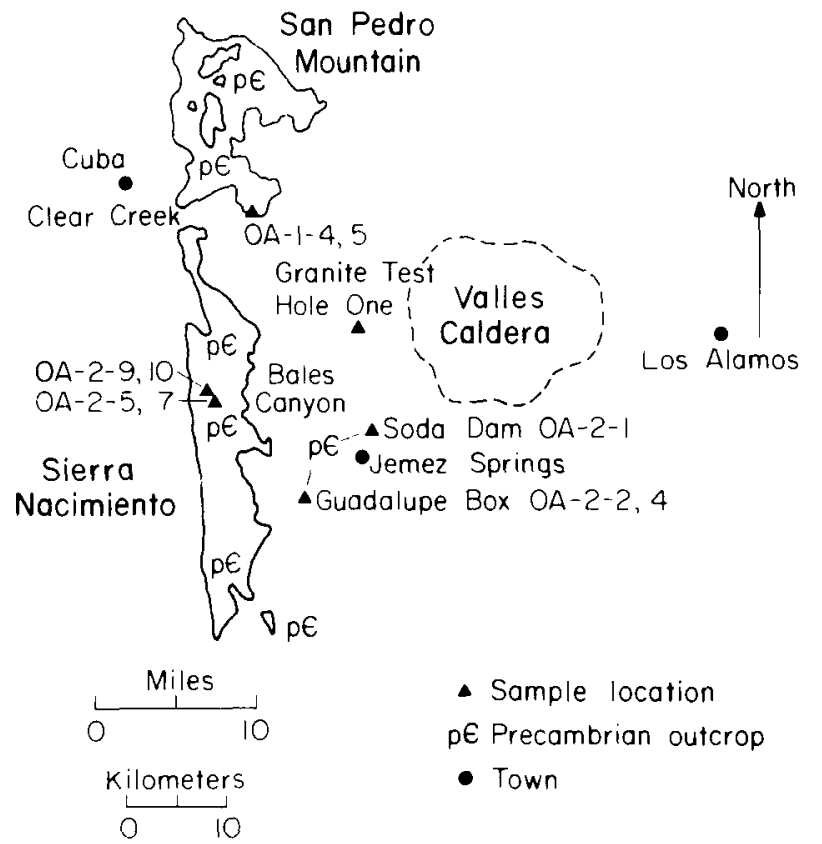

Fig. 1 .

Index map of sample locations and Precambrian outcrops in the vicinity of the proposed geothermal test site. Precambrian outcrops after Dane and Backman.'

\section{PETROGRAPHIC CONVENTIONS AND TECH- NIQUES}

\section{A. Rock Names}

The names of granitoid rocks are assigned on the basis of the triangular diagram, Fig. 2. The percentages of quartz $(\mathrm{Q})$, alkali feldspar (A), and plagioclase (P) are recalculated to 100 from the modal analysis.

Mineral modifiers preceding rock names are written in order of increasing abundance. Thus a "quartz-biotite amphibolite" has more biotite than quartz.

In this report, the terms amphibolite, aplite, and gneiss are used as follows:

- Amphibolite. Metamorphic rock composed mainly of plagioclase and hornblende; compositional term.

- Aplite. Fine- to medium-grained sugary veins of quartz and feldspar lacking dark minerals; textural term.

- Gneiss. Irregularly banded or foliated rock with alternating layers of light and dark, granular and micaceous minerals; textural term; adjective "gneissic."
TABLE II

\section{LOCATIONS OF SAMPLES FROM PRECAMBRIAN OUTCROPS}

\author{
Sandia Mountains
}

Samples transmitted to D. B. Slemmons by J. Rowley, letter of 10 May 1972.

1 "From the granite 'shield' of the north end of the Sandias; described by Orson [Anderson] as being "essentially a 500 foot thick mass of almost unjointed granite",

2 "Obtained about 100 feet away from sample \#1"

3 "Obtained from a road cut in Tijeras Canyon at the extreme southern end of the Sandia Mountains; described by Orson and observed by me to be highly jointed, both vertically and horizontally"

\section{San Pedro and Nacimiento Mountains}

Collected by P. Perkins, with assistance of $\mathrm{O}$. Anderson, F. West, and J. Balagna.

OA-1-4,5 Boulders in creek, Clear Creek Forest Camp, 2 miles west of Rio de Las Vacas on Highway 126. Same source as granite sent to the Haliburton Co. for hydrofracture experiment (D. Brown, personal communication, 1972)

OA-2-1 Roadcut on west side of Highway 4 at Soda Dam, north of Jemez Springs

OA-2-2 South side of southernmost tunnel in Guadalupe Box, canyon of Rio de Las Vacas

OA-2-4 North side of northernmost tunnel in Guadalupe Box, canyon of Rio de Las Vacas

OA-2-5,7 Bales Canyon, Forest Road 534, about 1 mile northwest of Rito la Cueva spring

OA-2-9,10 Nacimiento Mountains, Forest Road 534, 5.5 road miles west of Porter Landing junction

Thus, a "tonalite-aplite" has the mineralogy of a tonalite and the texture of an aplite. A "gneissic granite" has the texture of a gneiss and the mineralogy of a granite, and is of presumed igneous origin. A "granite gneiss" would have the mineralogy of a granite and the texture of a gneiss, with no presumed igneous origin. 


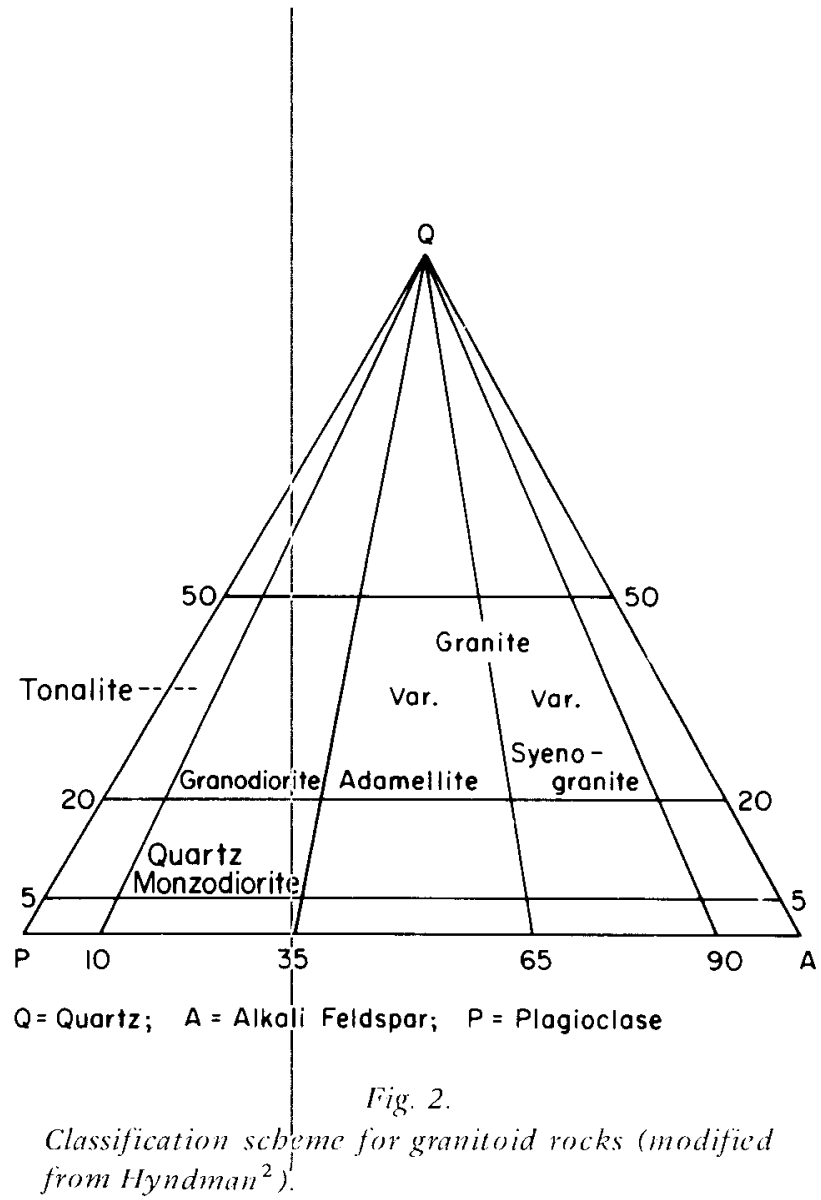

\section{B. Color}

Colors are described in accordance with the RockColor Chart of the Geological Society of America. ${ }^{3}$ Rock colors for hand specimens refer to wet sawed surfaces in the interior of core fragments.

\section{Grain Size}

Grain sizes refer to the following dimensions: finegrained, less than $1 \mathrm{~mm}$; medium-grained, 1 to $5 \mathrm{~mm}$; coarse-grained, $5 \mathrm{~mm}$ to $3 \mathrm{~cm}$.

\section{Texture}

Foliation refers to any planar or subplanar structure observable in hand specimen or thin section. The orientation of the foliation in the core fragments is referred to the axis of the core and the "up" (toward the surface) arrow marked on all specimens. The following diagram illustrates a foliation dip of $35^{\circ}$.

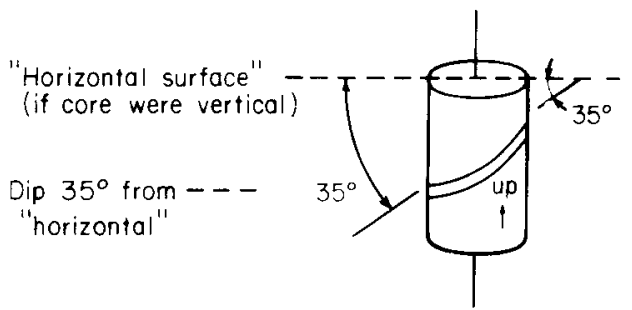

\section{E. Plagioclase Composition}

The composition of plagioclase was determined by universal stage measurements of twinned grains using the methods of Slemmons. ${ }^{4}$ Ambiguous cases were resolved by refractive index comparisons with Canada balsam and, where possible, measurements of Carlsbad or Carlsbadalbite twins and twins with a second set of cleavages.

\section{MODAL ANALYSES}

Modal analyses were made with a Swift automatic point counter. One analysis was made of each homogeneous thin section and of each distinctive layer in banded sections. Counting intervals were $1 / 3 \mathrm{~mm}$ horizontally and $2 / 3 \mathrm{~mm}$ vertically for the $\mathrm{PD}-2$ series, and $1 / 3 \mathrm{~mm}$ horizontally and vertically for the other slides. The number of counts per slide is indicated later in the summary tables. To test reproducibility, modal analyses of slides PD-2-b and PD-2-5 were performed twice (Table III). An average of the two analyses is recorded in the summary tablc.

A chart for evaluating point counting results has been presented by Van Der Plas and Tobi (Ref. 5, Fig. 1, p. 88). This chart is applicable to cases where the counting interval is larger than the largest grains included in the analysis. This is not the case for the medium-grained rocks discussed here. However, the chart may be consulted as an approximate indication of reliability. Results in the summary tables (Tables IV, V, and VI) represent values to the nearest percent. For trace minerals with abundance of less than $1 \%$, the actual number of counts is recorded in the tables.

\section{TABLE III}

\section{REPRODUCIBILITY OF MODAL ANALYSES}

(Values to the nearest percent)

\begin{tabular}{|c|c|c|c|c|}
\hline & $\begin{array}{c}\text { PD-2-b } \\
\text { First } \\
\text { Trial }\end{array}$ & $\begin{array}{c}\text { PD-2-b } \\
\text { Second } \\
\text { Trial }\end{array}$ & $\begin{array}{c}\text { PD-2-5 } \\
\text { First } \\
\text { Trial }\end{array}$ & $\begin{array}{c}\text { PD-2-5 } \\
\text { Second } \\
\text { Trial }\end{array}$ \\
\hline Quartz & 38 & 35 & 33 & 31 \\
\hline Microcline & 18 & 19 & 27 & 31 \\
\hline $\begin{array}{c}\text { Plagioclase } \\
\text { (Total) }\end{array}$ & 36 & 39 & 33 & 31 \\
\hline Biotite & 6 & 6 & 5 & 5 \\
\hline Accessories & 2 & 1 & 2 & 2 \\
\hline
\end{tabular}


Counts: Points counted; in cases where slides were analyzed twice, counts from both traverses were added together.

Act: actinolite

Al: allanite

Ap: apatite

$\mathrm{Bi}$ : biotite

Cc: calcite

Ch: chlorite

Cm: cummingtonite

Ep: epidote

$\mathrm{Hb}$ : hornblende

Mi: microcline (includes perthite films of plagioclase)

$\mathrm{Mu}$ : muscovite (discrete flakes larger than sericite in altered plagioclase)
My: myrmekite (probably intergrowth of quartz and oligoclase)

Op: opaque minerals: see also Table IX

$\mathrm{Pl}$ : plagioclase (includes antiperthite blebs of microcline and rims of albite): see also Table VII

Ps: altered plagioclase, generally sericitized: see Table VIII

Q: quartz

Sp: sphene

$\mathrm{Zi}$ : zircon

TABLE IVa

MODAL ANALYSES OF GRANITOID ROCKS ${ }^{\mathrm{a}}$

Granite Test Hole One

\begin{tabular}{|c|c|c|c|c|c|c|c|c|c|c|c|c|c|c|c|c|c|c|}
\hline $\begin{array}{c}\text { Thin } \\
\text { Section }\end{array}$ & $\begin{array}{c}\text { Depth } \\
\text { below } \\
2422 \mathrm{ft} \\
\text { (ft) }\end{array}$ & Counts & $\mathbf{B i}$ & $\mathbf{M i}$ & Pl & Ps & $\mathbf{Q}$ & Al & $\mathbf{A p}$ & $\mathrm{Cc}$ & Ch & $\mathbf{E p}$ & $\mathbf{H b}$ & $\mathbf{M u}$ & $\mathbf{M y}$ & $\mathrm{Op}$ & $\mathrm{Sp}$ & $\mathrm{Zi}$ \\
\hline PD-2-a & 0 & 2274 & 4 & 32 & 20 & 3 & 39 & & $5 c$ & $2 c$ & $4 c$ & & & $1 c$ & 1 & $12 \mathrm{c}$ & & \\
\hline PD-2-b & 0 & 4631 & 6 & 18 & 37 & 1 & 37 & & $4 c$ & $1 \mathrm{c}$ & $4 c$ & & & $1 \mathrm{c}$ & $12 \mathrm{c}$ & 1 & & \\
\hline PD-2-c & 0 & 2040 & 8 & 21 & 29 & 1 & 39 & & $6 c$ & $1 c$ & $5 c$ & & & & 1 & 1 & $1 \mathrm{c}$ & $1 \mathrm{c}$ \\
\hline PD-2-1 & 8 & 2548 & 2 & 28 & 18 & 8 & 40 & & $2 c$ & & 1 & $7 c$ & & $8 c$ & $2 c$ & 1 & $3 c$ & \\
\hline PD-3-5 & 16 & 2616 & 6 & 40 & 19 & 6 & 27 & & $4 c$ & $2 c$ & 1 & & & $6 c$ & $12 c$ & 1 & $16 \mathrm{c}$ & \\
\hline PD-2-2 & 22 & 2296 & 6 & 35 & 21 & 13 & 20 & & $8 c$ & $13 c$ & $11 \mathrm{c}$ & $5 c$ & & $16 \mathrm{c}$ & $6 c$ & 1 & $17 c$ & $1 c$ \\
\hline matrix & 22 & 1016 & 10 & 21 & 32 & 18 & 13 & & $4 c$ & $8 c$ & $3 c$ & $2 c$ & & $5 c$ & $9 \mathrm{c}$ & $9 c$ & 2 & $1 \mathrm{c}$ \\
\hline aplite & 22 & 565 & & 52 & 6 & 3 & 39 & & & & & & & & & & & \\
\hline PD-2-3 & 45 & 2268 & 4 & 37 & 20 & 8 & 28 & $2 c$ & $3 c$ & $3 c$ & $6 c$ & $5 c$ & & $6 c$ & $9 c$ & 1 & $6 c$ & \\
\hline PD-2-4 & 59 & 2583 & 6 & 30 & 27 & 3 & 31 & & $8 c$ & $4 c$ & $11 c$ & $1 \mathrm{c}$ & & $9 \mathrm{c}$ & $10 \mathrm{c}$ & 1 & 1 & \\
\hline PD-2-5 & 62 & 5445 & 5 & 29 & 29 & 3 & 32 & & $3 c$ & $8 c^{\circ}$ & $12 \mathrm{c}$ & & & $14 c$ & $16 c$ & $8 c$ & & $1 \mathrm{c}$ \\
\hline matrix & 62 & 986 & 8 & 22 & 39 & 1 & 29 & & $2 c$ & & & & $1 \mathrm{c}$ & $2 c$ & $4 c$ & $2 c$ & & \\
\hline PD-3-7 & 75 & 1688 & 13 & 15 & 27 & 10 & 32 & & $2 c$ & & $8 c$ & $2 c$ & & $2 c$ & 1 & 1 & & \\
\hline PD-2-6 & 79 & 2197 & 8 & 24 & 32 & 11 & 22 & & & $8 c$ & $5 c$ & $2 c$ & & 1 & $10 c$ & 1 & $7 c$ & \\
\hline matrix & 79 & 1021 & 15 & 2 & 51 & 4 & 26 & & $2 c$ & $3 c$ & $3 c$ & & & $2 c$ & $2 c$ & $8 c$ & $1 \mathrm{c}$ & \\
\hline PD-2-7 & 80 & 2185 & 21 & 10 & 38 & 1 & 27 & & $7 c$ & & $1 \mathrm{c}$ & $1 \mathrm{c}$ & & $1 c$ & 1 & 1 & $6 c$ & \\
\hline PD-2-8 & 88 & 2256 & 14 & 13 & 43 & 4 & 23 & & $3 c$ & & $3 \mathrm{c}$ & $1 \mathrm{c}$ & & $1 \mathrm{c}$ & 1 & 1 & $4 c$ & 10 \\
\hline PD-2-12 & 93 & 2028 & 13 & 10 & 32 & 14 & 26 & & $4 c$ & $4 c$ & 1 & 1 & & $3 c$ & $9 c$ & $6 \mathrm{c}$ & $5 c$ & $1 \mathrm{c}$ \\
\hline PD-3-6 & 94 & 2432 & 11 & 7 & 12 & 35 & 30 & & $12 c$ & $1 \mathrm{c}$ & $5 c$ & $15 c$ & & 1 & 2 & $7 c$ & 1 & $1 c$ \\
\hline
\end{tabular}

${ }^{\mathrm{a}}$ In last 16 columns: $\mathrm{c}=$ actual counts; other numbers are percentages 
TABLE IVl,

MODAL ANALYSES OF (;RANITOID ROCKS'

\begin{tabular}{|c|c|c|c|c|c|c|c|c|c|c|c|c|c|c|c|c|}
\hline $\begin{array}{c}\text { Thin } \\
\text { Section }\end{array}$ & Counts & $\mathrm{Bi}$ & Mi & PI & Ps & $Q$ & $A_{p}^{\prime}$ & $C c$ & Ch & lip & $\mathrm{Hb}$ & $\mathbf{M u}$ & My & $O_{p}$ & sp & $Z i$ \\
\hline $1 \mathrm{~b}$ & 2258 & & 44 & 1 & 33 & 16 & & & & & & & & & & \\
\hline $2^{b}$ & 2306 & & 18 & 1 & 38 & 34 & $5 c$ & & + & $1 c$ & $x^{\circ}$ & & & 1 & & \\
\hline $3^{b}$ & 2943 & 15 & 16 & 7 & 12 & 39 & $6 c$ & $2 c$ & 0 & & 1 & & & 1 & 60 & \\
\hline $\mathrm{OA}-1-4^{\mathrm{C}}$ & 1557 & 5 & 19 & 3 & 29 & 42 & 1 & & $8 c$ & 1 & 30 & & 1 & 2 & 6 & 16 \\
\hline $\mathrm{OA}-1-5^{\mathrm{C}}$ & 2694 & 4 & 25 & 4 & 44 & 19) & $2 c$ & & 20 & 3 & $2 c$ & & & $2 c$ & & \\
\hline $\mathrm{OA}-2-1^{\mathrm{d}}$ & 1849 & & 11 & $4 c$ & 57 & 29 & $2 c$ & & $1+i$ & t & & $+c$ & & & $7 c$ & \\
\hline$O \wedge-2-2^{\mathrm{e}}$ & $27+1$ & 8 & 18 & 23 & 25 & 18 & $1 c$ & & 40 & & & 2 & & $1 c$ & 10 & \\
\hline $\mathrm{OA}-2-4^{\mathrm{e}}$ & 2434 & 1 & 49 & 1 & 25 & 15 & 11 & $7 c$ & $12 c$ & 1 & 3 & & $1 c$ & 1 & 1 & \\
\hline ()A $-2-5^{f}$ & 2832 & 4 & 44 & 8 & 17 & 27 & $5 c$ & & 5 & 12 & $5 i$ & & $3 c$ & 1 & 2 & \\
\hline $\mathrm{OA}-2-7^{\mathrm{f}}$ & 2039 & 3 & 39 & 7 & 24 & 27 & & & $4 c$ & $2 c$ & & 1 & $3 c$ & $1 c$ & & \\
\hline
\end{tabular}

an last 15 columns: $c$ = actual count; other numbers are perecntages dSoda Dam

bandia Mountains

San Pedro Mountain

CGuadalupe Box

${ }^{f}$ Nacimiento Mountains

TABLEV

MODAL ANALYSES OI: AMPHIBOLITES ANI) APLITES

Granite Test Hole One

\begin{tabular}{|c|c|c|c|c|c|c|c|c|c|c|c|c|c|c|c|c|c|c|}
\hline $\begin{array}{c}\text { Thin } \\
\text { Section }\end{array}$ & $\begin{array}{c}\text { Depth } \\
\text { below } \\
2422 \mathrm{ft} \\
\text { (ft) }\end{array}$ & Counts & Act & $\mathrm{Bi}$ & $\mathrm{Cm}$ & $\mathrm{Hb}$ & Pl & $P_{s}$ & $Q$ & Ap & $\mathrm{Cc}$ & $\mathrm{Ch}$ & $\mathbf{E p}$ & $\mathbf{M i}$ & $\mathbf{M u}$ & $O_{p}$ & $s_{p}$ & $Z \mathrm{i}$ \\
\hline \multicolumn{19}{|l|}{ P'D-2-13 } \\
\hline light band & 96 & 950 & & 15 & & 8 & 36 & 2 & 36 & & & & $G$ & 2 & & $3 i$ & $1 c$ & $1 c$ \\
\hline dark band & 96 & 345 & & 16 & & 42 & 26 & 3 & 12 & & & & $2 \mathrm{c}$ & $1 \mathrm{c}$ & & & $1 \mathrm{c}$ & \\
\hline aplite & 96 & 527 & & 5 & & 1 & +2 & 6 & 41 & & & $1 c$ & $3 c$ & 4 & & $3 c$ & & \\
\hline PD-2-1 4 & 98 & 2303 & & 9 & & 61 & 21 & 6 & & $1 c$ & 2 & $4 c$ & $15 c^{\circ}$ & & & $4 c$ & 1 & \\
\hline PD-3-8 & 98 & 1952 & & 17 & & +4 & 20 & 16 & 1 & $2 c$ & $3 c$ & $7 \mathrm{c}$ & $7 c$ & & & $3 c$ & & \\
\hline PD-2-15 & 100 & 2205 & & 14 & & 18 & 15 & 26 & 24 & $5 c$ & $3 c$ & 1 & $160^{\circ}$ & & $1 \mathrm{c}$ & $1 c$ & 1 & \\
\hline matrix & 100 & 381 & & 22 & & 42 & 13 & 13 & 5 & $2 c$ & & 2 & $2 c$ & & & $1 c$ & 2 & \\
\hline aplite & 100 & 727 & & 3 & & 3 & 19 & 38 & 36 & & & $2 c$ & & & & & & \\
\hline PD-2-9 & 105 & 2276 & & 15 & & 46 & 19 & 11 & 8 & $1 c$ & & $6 c$ & & & & 9 & $120^{\circ}$ & \\
\hline \multicolumn{19}{|l|}{ PD-2-11 } \\
\hline matrix & 108 & 1192 & & 22 & & 44 & 20 & 11 & 1 & $3 c$ & 1 & $3 c$ & $1 c$ & & $1 c$ & & 1 & \\
\hline aplite & 108 & 676 & & 7 & & 1 & 18 & 35 & 39 & & & & & & & & & \\
\hline \multicolumn{19}{|l|}{ PD-2-10 } \\
\hline matrix & 108 & 1033 & & 56 & & 5 & 35 & & 2 & & $3 c$ & & & & & 16 & $5 c$ & \\
\hline aplite & 108 & 593 & & 7 & & 38 & 11 & & 40 & & 4 & & & & & & & \\
\hline PD-2-16 & 120 & 2495 & & 11 & & 55 & 20 & 13 & & $2 c$ & $1 c$ & $15 \mathrm{c}$ & 50 & & & $1 c$ & $11 c$ & \\
\hline$P D-3-1 b$ & 127 & 2192 & 26 & & & & 12 & 35 & $8 c$ & & 7 & 18 & 120 & & & & 1 & \\
\hline PD-3-3 & 135 & 3003 & & 20 & & 12 & 23 & 11 & 34 & $2 c$ & $8 c$ & $8 c$ & $1 c$ & & & $6 c$ & $7 c$ & $2 c$ \\
\hline matrix & 127 & 889 & & 10 & & 39 & 21 & 22 & 6 & $2 c$ & $4 c$ & $4 c$ & IC & & & $1 c$ & $3 i$ & \\
\hline $\begin{array}{l}\text { aplite } \\
\text { biotite }\end{array}$ & 127 & 1167 & & 5 & & & 16 & 8 & 70 & & 10 & $3 c$ & & & & & $1 c$ & $2 c$ \\
\hline band & 127 & 947 & & 47 & & & 34 & 3 & 15 & & & $1 c$ & & & & $5 i$ & $3 c$ & \\
\hline PD-3-2 & 137 & 1084 & & 29 & & 28 & 19 & 12 & 10 & $2 c$ & $4 c$ & $1 c$ & $2 c$ & & & & 1 & \\
\hline PD-3-4 & 152 & 1375 & & 15 & & 29 & 24 & 14 & 14 & $3 c$ & $1 c$ & 1 & $4 c$ & & & $1 c$ & 1 & 10 \\
\hline OA-2-9 & --- & 2685 & & & & 54 & 29 & 14 & 2 & $1 c$ & & & & & & 1 & & \\
\hline$O A-2-10$ & --- & 2326 & & 1 & 18 & 73 & 7 & 1 & & & $1 c$ & & & & & $3 c$ & $9 c$ & \\
\hline
\end{tabular}

an last 9 columns: $\mathrm{c}=$ actual counts; other numbers are percentages

${ }^{b}$ Not amphibolite, but chlorite-actinolite-plagioclase rock 
TABLE VIa

\section{CRYSTAL CONTACTS ${ }^{a}$}

Granites

\begin{tabular}{|c|c|c|c|c|c|c|c|c|c|c|c|c|c|c|}
\hline $\begin{array}{l}\text { Sample } \\
\text { Crystals }\end{array}$ & PD-2-c & PD-3-5 & PD-3-7 & PD-3-6 & 1 & 2 & 3 & OA-1-4 & OA-1-5 & OA-2-1 & OA-2-2 & OA-2-4 & $\mathrm{OA}-2-5^{\mathrm{b}}$ & OA-2-7 \\
\hline $\mathrm{Bi}-\mathrm{Bi}$ & $3 c$ & $5 \mathrm{c}$ & $4 c$ & $5 \mathrm{c}$ & & & $5 c$ & $4 c$ & $7 \mathrm{c}$ & & $6 c$ & & & \\
\hline $\mathrm{Bi}-\mathrm{Mi}$ & $4 c$ & $2 \mathrm{c}$ & $1 \mathrm{c}$ & $1 \mathrm{c}$ & & & $2 \mathrm{c}$ & $1 \mathrm{c}$ & & & & & & \\
\hline $\mathrm{Bi}-\mathrm{Pl} / \mathrm{Ps}$ & 8 & 9 & 13 & 7 & & & $1 \mathrm{c}$ & & & & 6 & & & $1 \mathrm{c}$ \\
\hline Bi-Q & 5 & 7 & 7 & 5 & & & 9 & $4 c$ & $4 c$ & & 8 & & $6 c$ & $1 \mathrm{c}$ \\
\hline $\mathrm{Mi}-\mathrm{Mi}$ & 4 & $1 \mathrm{c}$ & 4 & $1 \mathrm{c}$ & 4 & $1 c$ & $1 c$ & & & & $1 \mathrm{c}$ & $1 \mathrm{c}$ & $1 \mathrm{c}$ & $1 c$ \\
\hline $\mathrm{Mi}-\mathrm{P} \mathrm{l} / \mathrm{Ps}$ & & & & & 9 & 8 & & & $1 \mathrm{c}$ & & & 20 & 8 & $3 c$ \\
\hline $\mathrm{Mi}-\mathrm{Q}$ & 7 & 14 & 9 & $2 c$ & 14 & 14 & 8 & 4 & 9 & $1 c$ & & $2 \mathrm{c}$ & 5 & $7 \mathrm{c}$ \\
\hline $\mathrm{Pl} / \mathrm{Ps}-\mathrm{Pl} / \mathrm{Ps}$ & 20 & & $2 c$ & 32 & 7 & & $2 c$ & & & 73 & & 13 & 7 & $1 \mathrm{c}$ \\
\hline Pl/Ps-Q & 18 & 13 & 20 & 7 & $1 \mathrm{c}$ & 5 & & & 11 & 10 & 14 & 5 & $9 c$ & $6 c$ \\
\hline $\mathrm{Q}-\mathrm{Q}$ & 16 & 14 & 13 & 40 & 51 & 52 & 30 & 70 & 54 & 12 & 27 & & 14 & $11 \mathrm{c}$ \\
\hline Cracks $^{b}$ & & & & & & & & & & & & & 58 & 92 \\
\hline [Footnote c] & 9 & 3 & 5 & 13 & 3 & 3 & 4 & 9 & 5 & 19 & 4 & 3 & 10 & 35 \\
\hline
\end{tabular}

$a_{c}=$ number of counts for uncommon pairs; other numbers are percentages of contacts

${ }^{\mathrm{b}}$ Samples OA-2-5 and OA-2-7 are weathered and have begun to disintegrate along grain boundaries, leaving open cracks in thin section

${ }^{c_{P}}$ Percentage of total modal counts represented by crystal contacts or open cracks encountered in analysis 
TABLE VIb

\title{
CRYSTAL CONTACTS ${ }^{\mathrm{a}}$
}

\author{
Amphibolites
}

\begin{tabular}{|c|c|c|c|c|c|c|}
\hline $\begin{array}{l}\text { Sample } \\
\text { Crystals }\end{array}$ & PD-3-8 & PD-3-3 & PD-3-2 & PD-3-4 & OA-2-9 & $O A-2-10$ \\
\hline $\mathrm{Bi}-\mathrm{Bi}$ & 5 & $1 \mathrm{c}$ & $2 \mathrm{c}$ & 15 & & \\
\hline $\mathrm{Bi}-\mathrm{Cm}$ & & & & & & $2 c$ \\
\hline $\mathrm{Bi}-\mathrm{Hb}$ & 22 & $5 c$ & 7 & & & $3 c$ \\
\hline $\mathrm{Bi}-\mathrm{P}] / \mathrm{Ps}$ & 13 & 11 & 15 & 43 & & $2 c$ \\
\hline $\mathrm{Bi}-\mathrm{Q}$ & $2 \mathrm{c}$ & 5 & 19 & 26 & & \\
\hline $\mathrm{Cm}-\mathrm{Cm}$ & & & - & & & 8 \\
\hline $\mathrm{Cm}-\mathrm{Hb}$ & & & & & & 22 \\
\hline $\mathrm{Cm}-\mathrm{Pl} / \mathrm{Ps}$ & & & & & & 8 \\
\hline $\mathrm{Hb}-\mathrm{Hb}$ & 17 & 22 & 10 & & 40 & 50 \\
\hline $\mathrm{Hb}-\mathrm{Pl} / \mathrm{Ps}$ & 36 & 33 & 27 & & 50 & 11 \\
\hline $\mathrm{Hb}-\mathrm{Q}$ & & $3 c$ & 8 & & $6 c$ & \\
\hline $\mathrm{Pl} / \mathrm{Ps}-\mathrm{Pl} / \mathrm{Ps}$ & $5 c$ & $4 c$ & $4 c$ & $5 c$ & 8 & $1 \mathrm{c}$ \\
\hline Pl/Ps-Q & & 12 & $2 \mathrm{c}$ & 8 & $2 c$ & \\
\hline$Q-Q$ & & $3 c$ & $1 \mathrm{c}$ & & & \\
\hline [Footnote b] & 15 & 13 & 10 & 15 & 15 & 19 \\
\hline
\end{tabular}

\section{CRYSTAL CONTACTS}

During the proposed geothermal experiment, hot water will be circulated in Precambrian rocks at depth. Because circulating fluids are likely to pass along grain boundaries, a study of the frequency of contacts between crystals was made in the course of modal analysis.

During the analysis, as the slide is automatically advanced, the intersection of the cross-hairs usually falls within a single grain. In this case, the identity of the grain is noted and added to the modal count. When the crosshairs fall on the contact between two grains, the following convention is observed. A note is made of the identity of the two touching crystals, and the crystal being approached as the slide is advanced is counted toward the mode. In this manner, simultaneous records are kept of grains in the modal count and frequency and identity of crystalcrystal contacts.
TABLE VII

PLAGIOCLASE COMPOSITION

\begin{tabular}{|c|c|c|}
\hline Sample & Rock & (\%) \\
\hline PD-2-a & gneissic adamellite & 23 \\
\hline$P D-2-b$ & gncissic adamellite & $24-26$ \\
\hline PD-2-c & gneissic adamellite & 24 \\
\hline PD-2-1 & gneissic adamellite & 24 \\
\hline PD-2-2 & $\begin{array}{l}\text { adamellite veined by syeno- } \\
\text { granite-aplite/pegmatite }\end{array}$ & $24-26$ \\
\hline PD-2-4 & $\begin{array}{l}\text { adamellite } \\
\text { albite rims on oligoclase }\end{array}$ & $\begin{array}{c}24-26 \\
3-5\end{array}$ \\
\hline $\mathrm{PD}-2-5$ & $\begin{array}{l}\text { gncissic granodiorite matrix } \\
\text { adamellite-aplite }\end{array}$ & $\begin{array}{c}30 \\
28-30\end{array}$ \\
\hline PD-2-7 & gneissic granodiorite & $25-28$ \\
\hline PD-2-8 & gneissic granodiorite & 29 \\
\hline PD-2-12 & gneissic granodiorite & $25-29$ \\
\hline PD-2-13 & $\begin{array}{l}\text { quartz-biotite amphibolite } \\
\text { biotite-quartz-oligoclase } \\
\text { gneiss } \\
\text { tonalite-aplite }\end{array}$ & $\begin{array}{c}26 \\
27-30\end{array}$ \\
\hline PD-2-15 & $\begin{array}{l}\text { biotite-amphibolite } \\
\text { tonalite-aplite }\end{array}$ & $27-31$ \\
\hline PD-2-11 & $\begin{array}{l}\text { biotite-amphibolite } \\
\text { tonalite-aplite }\end{array}$ & $\begin{array}{l}30 \\
30\end{array}$ \\
\hline PD-2-16 & biotite-amphibolite & $34-38$ \\
\hline
\end{tabular}


TABLE VIII ALTERATION OF PLAGIOCLASE [Ps/(PI+Ps)] AND
Q-A-P VALUES (Q, Mi, PI+Ps = 100) CALCULATED
FROM MODAL ANALYSES BEFORE ROUNDING

\begin{tabular}{|c|c|c|c|c|c|}
\hline $\begin{array}{c}\text { Thin } \\
\text { Section }\end{array}$ & $\begin{array}{c}\text { Alteration } \\
\text { of Plagioclase } \\
(\%)\end{array}$ & $\begin{array}{c}Q \\
(\%)\end{array}$ & $\begin{array}{c}A \\
(\%)\end{array}$ & $\begin{array}{c}\mathrm{P} \\
(\%)\end{array}$ & $\begin{array}{c}A \\
A+P \\
(\%)\end{array}$ \\
\hline PD-2-a & 13 & 41 & 34 & 25 & 0.58 \\
\hline PD-2-b & 3 & 40 & 20 & 40 & 0.33 \\
\hline PD-2-c & 5 & 43 & 23 & 34 & 0.41 \\
\hline PD-2-1 & 31 & 44 & 27 & 29 & 0.49 \\
\hline PD-3-5 & 23 & 29 & 43 & 28 & 0.61 \\
\hline \multicolumn{6}{|l|}{ PD-2-2 } \\
\hline matrix & 35 & 15 & 26 & 59 & 0.30 \\
\hline aplite & 33 & 39 & 53 & 8 & 0.87 \\
\hline PD-2-3 & 28 & 31 & 40 & 29 & 0.58 \\
\hline PD-2-4 & 10 & 35 & 33 & 32 & 0.51 \\
\hline PD-2-5 & 10 & 34 & 31 & 35 & 0.48 \\
\hline matrix & 2 & 32 & 24 & 44 & 0.35 \\
\hline PD-3-7 & 27 & 39 & 18 & 43 & 0.29 \\
\hline PD-2-6 & 3 & 28 & 31 & 41 & 0.43 \\
\hline matrix & 8 & 31 & 2 & 67 & 0.03 \\
\hline PD-2-7 & 4 & 35 & 13 & 52 & 0.21 \\
\hline PD-2-8 & 8 & 27 & 15 & 58 & 0.20 \\
\hline PD-2-12 & 45 & 32 & 12 & 56 & 0.18 \\
\hline PD-3-6 & 75 & 36 & 8 & 56 & 0.15 \\
\hline \multicolumn{6}{|l|}{ PD-2-13 } \\
\hline light band & 5 & & & & \\
\hline dark band & 11 & & & & \\
\hline aplite & 13 & 44 & 4 & 52 & 0.07 \\
\hline
\end{tabular}

\section{OPAQUE MINERALS}

Polished sections were prepared from approximately one half of the samples from Granite Test Hole One. The results of preliminary examination in reflected light are shown in Table IX.

\section{DISCUSSION}

This study describes 25 samples (27 thin sections) from $152 \mathrm{ft}$ of drill core taken from Granite Test Hole One, and 12 examples of Prccambrian rocks from the region of the proposed geothermal test site.

The specimens fall into two groups: granitoid rocks and amphibolites. Such rocks are typical constituents of
TABLE IX

\section{OPAQUE MINERALS}

(Listed in order of decreasing abundance)

\begin{tabular}{|c|c|c|}
\hline $\begin{array}{l}\text { Sample } \\
\text { Number }\end{array}$ & $\begin{array}{c}\text { Depth } \\
\text { below } \\
2422 \mathrm{ft} \\
(\mathrm{ft})\end{array}$ & Mineralogy \\
\hline PD-2-abc & 0 & $\begin{array}{l}\text { magnetite, ilmenite with minute } \\
\text { exsolution lamellae of hematite }\end{array}$ \\
\hline PD-3-5 & 16 & magnetite, pyrite, chalcopyrite \\
\hline PD-2-2 & 22 & magnetite, pyrite, chalcopyrite \\
\hline PD-2-3 & 45 & magnetite, pyrite \\
\hline PD-2-4 & 59 & magnetite, pyrite \\
\hline PD-3-7 & 75 & magnetite \\
\hline PD-2-8 & 88 & magnetite \\
\hline PD-3-6 & 94 & pyrite \\
\hline PD-3-1 & 127 & magnetite, pyrite, chalcopyrite \\
\hline PD-3-3 & 135 & magnetite, pyrite \\
\hline PD-3-4 & 152 & pyrite, chalcopyrite \\
\hline
\end{tabular}

the Precambrian terrains of many areas of the world. The compositions of granitoid rocks are shown in Figs. 3 and 4. Compositions are plotted in terms of the Q-A-P classification. The sequence of rock types in Granite Test Hole One is shown in Table $X$.

The core in Precambrian crystalline rocks consists of two major units. The upper unit (about $94 \mathrm{ft}$ ) consists of medium- to coarse-grained, brownish-gray granitoid rocks with variable amounts of pale red microcline and texture varying from typical granitic (massive, hypidiomorphic granular) to gneissic (alternating bands of quartz-feldspar and biotite) with megacrysts of microcline. The lower unit (about $61 \mathrm{ft}$ in the interval sampled) consists of fine-grained, dark-gray biotite-amphibolite veined by medium-grained, light-gray aplite. The upper $3 \mathrm{ft}$ of the amphibolite is foliated, probably parallel to the foliation of the overlying granodiorite gneiss. Near the contact with the granodiorite, the amphibolite contains accessory microcline. 


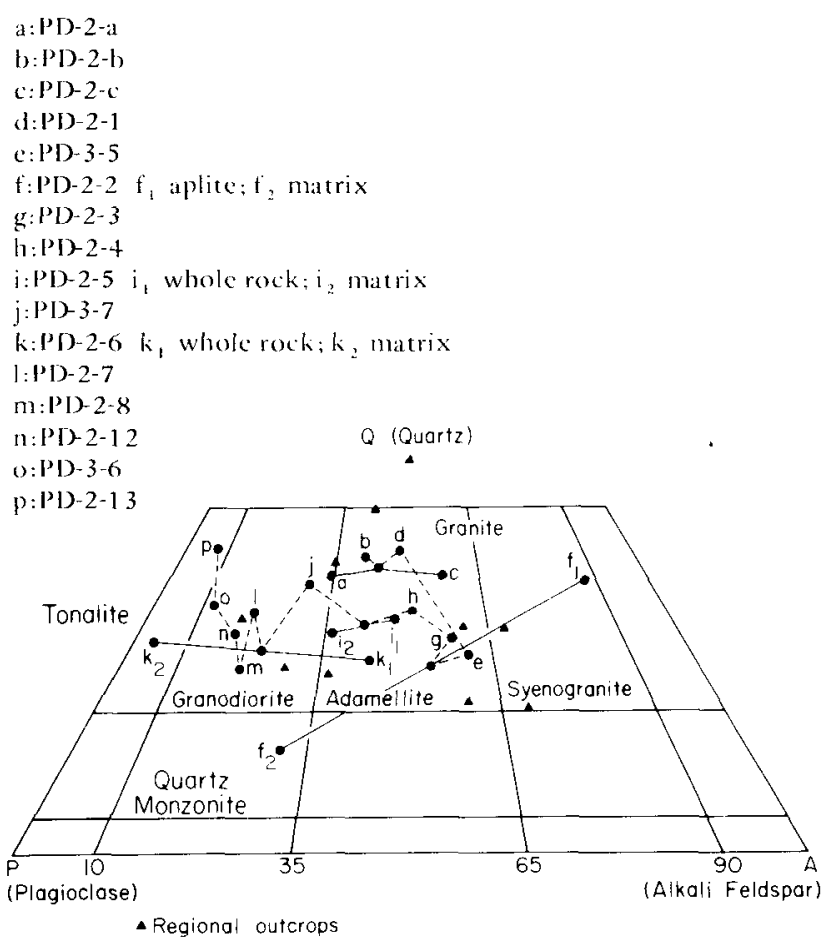

lig. 3.

Compositions of granitold rocks, Gramito Test Hole' One and regional onterops.

In granitoid rocks the variability of the core is due largely to variable microcline content. Microcline is ubiquitous $(30-40 \%)$ in the upper $60 \mathrm{ft}$, but is spotty in the 2484- to 2501-ft interval (in clusters of megacrysts and in adamellite-aplite) and constitutes $10 \%$ of the rock in the lower $13 \mathrm{ft}$ of the granitoid mass. The texture of the granitoid unit is gneissic at the top (with augen of microcline) and at the bottom (defined by abundant biotite), and massive in the middle $40 \mathrm{ft}$. As microcline content decreases, biotite content increases with depth from $5 \%$ in the upper augengneissic granite to $20 \%$ in the lower gneissic granodiorite, as the amphibolite is approached.

Because a hydrofracturing experiment is to be conducted in these rocks, special attention was paid to cracks which occurred before or during the process of thinsectioning. All cracks observed in thin section are along thin calcite veins. These veins are both parallel to and transverse to the foliation of the rocks. No cracks were found in thin section independent of calcite, but not all calcite veins are associated with cracks. In hand specimen, cracks parallel to calcite veins and parallel to chloritecoated surfaces are present.

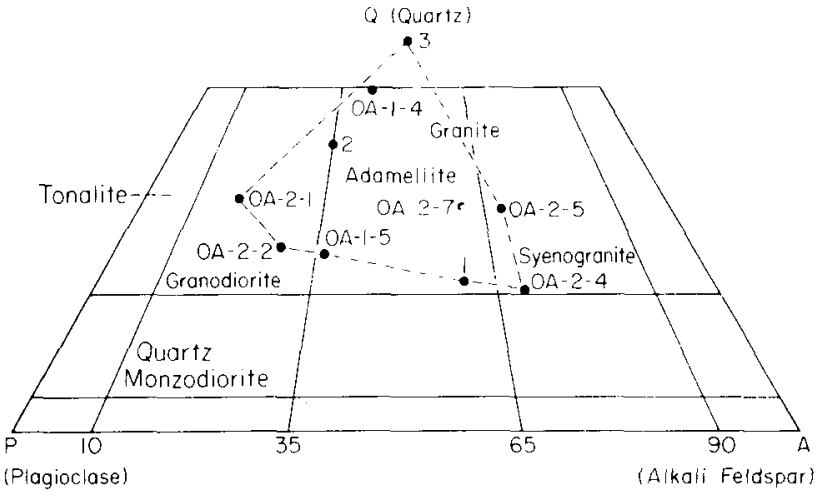

lig. 4 .

Compositions of granitost rocks: Samdia Mommtains (1, 2, 3), San Pedro Monmtain (OA-1-4, 5), Soda Dam (OA-2-1), Gutrdalupe Box (OA-2-2, 4), Nacimicuto Motritaims $(0)-2-5,7)$.

Bochm lamellac (planes of tiny tluid inclusions, some with moveable bubbles) are present in much of the quart of the granitoid rocks. The lamellac are continuous across grain boundaries. One set of lamellate is approximately parallel to the foliation; in some sections a second set appears in thin section at approximately right angles to the first. Quartz in the core almost always shows undulatory extinction.

Preliminary observations of the structure of the core rocks suggest that the foliation, where present, dips, on the average, $45^{\circ}$ relative to the core axis. The direction of strike of the foliation is not known. However, the dip of the foliation of the granodiorite gneiss and the foliation of the underlying biotite-amphibolite (and the dip of aplite veins in the amphibolite) are similar. If the foliations of these two rock types have the same strike, the structures in both rock types have a similar attitude. Calcite veins in hand specimen have two dominant orientations: parallel to the foliation and parallel to the axis of the core.

It is impossible to predict with certainty what sequence of rocks underlies the cored interval. However, preliminary field reconnaissance of surface Precambrian exposures suggests that granitic rock types are most abundant and amphibolite is of local extent. Although a large portion of Ciranite Test IIole One is in amphibolite (64 ft out of $155 \mathrm{ft}$ total), it is likely that granitic rocks are dominant at depth.

Robert L. Snith (personal communicarion, August 22, 1972) has observed in his studies of the Tertiary volcanics of the Jemez Mountains region that "red granite" is the 
TABLE X

\section{SEQUENCE OF ROCK TYPES IN GRANITE TEST HOLE ONE}

\begin{tabular}{|c|c|c|c|}
\hline $\begin{array}{c}\text { Depth } \\
(\mathrm{ft})\end{array}$ & $\begin{array}{l}\text { Interval } \\
\text { Between } \\
\text { Samples } \\
\quad(\mathrm{ft}) \\
\end{array}$ & $\begin{array}{c}\text { Thin } \\
\text { Section }\end{array}$ & Rock Type \\
\hline 2422 & & PD-2-a & \\
\hline & (8) & & Augengneissic granite, variety adamellite \\
\hline 2430 & & PD-2-1 & \\
\hline 2438 & & PD-3-5 & \\
\hline 2481 & (43) & PD-2-4 & Granite, variety adamellite \\
\hline $\begin{array}{l}2484 \\
2501\end{array}$ & (17) & $\begin{array}{l}\text { PD-2-5 } \\
\text { PD-2-6 }\end{array}$ & $\begin{array}{l}\text { Gneissic granite, variety adamellite; average composition } \\
\text { resulting from isolated clots of microcline megacrysts in } \\
\text { matrix ranging from gneissic granodiorite to gneissic } \\
\text { tonalite }\end{array}$ \\
\hline 2502 & & PD-2-7 & \\
\hline 2516 & (14) & PD-3-6 & Gneissic biotite-granodiorite \\
\hline 2518 & & PD-2-13 & $\begin{array}{l}\text { Biotite-amphibolite with minor microcline; tonalite-aplite } \\
\text { veins }\end{array}$ \\
\hline 2520 & $(29)$ & PD-2-14 & Biotite-amphibolite; tonalite-aplite veins \\
\hline 2549 & $(25)$ & PD-3-1 & Chlorite-actinolite-plagioclase rock \\
\hline 2574 & & PD-3-3 & Biotite-amphibolite; tonalite-aplite veins \\
\hline
\end{tabular}

Bottom of hole

dominant type of basement xenolith included in the pyroclastic rocks. Samples PD-2-2, 3, and 4 of this study (in the middle massive granite unit) are similar to the inclusions in the volcanics, according to Smith. He estimates that perhaps $95 \%$ of the xenoliths are "red granite," and he does not recall seeing amphibolite xenoliths. If amphibolites and metasedimentary rocks are a significant proportion of the basement, they should be more abundantly represented in the inclusion suite. On this basis, it is suggested that the amphibolite, which represents a considerable portion of the core, is a local septum in a dominantly granitoid basement.

A comparison of Figs. 3 and 4 shows that samples of regional Precambrian rocks resemble some of the rocks of the core in terms of quartz and feldspar ratios. The suite of rock types from Granite Test Hole One provides a good representation by fresh samples of regional rocks, with the exception of varieties unusually high in quartz or microcline content.

Hornblende-bearing granitoid rocks are represented in the regional samples (Table IVb) but are not present in the core. Also, an amphibolite with coexisting hornblende and cummingtonite (each with exsolution lamellae of the other, sample OA-3-10) was collected in the Nacimiento Mountains. This rock type is not present in the core.

The study of frequency of crystal-crystal contacts shows that, in granitoid rocks, the most common pairs, in decreasing order of abundance, are quartz-quartz, quartzplagioclase, microcline-quartz, and biotite-quartz. In amphibolites, the most common pairs are, in decreasing order, hornblende-plagioclase, hornblende-hornblende, biotite-plagioclase, and biotite-hornblende. 


\section{ACKNOWLEDGMENTS}

The author thanks Hatten S. Yoder, Jr., David B. Slemmons, and other members of the Los Alamos Geosciences Advisory Panel who guided and assisted this study. Laboratory facilities were provided at the University of California, Los Angeles, by Orson L. Anderson. Samples were obtained with the help of Orson L. Anderson and the following members of the Los Alamos Scientific Laboratory staff: John P. Balagna, Donald W. Brown, Robert M. Potter, and Francis G. West.

This work was performed while the author was a Visiting Staff Member of the Los Alamos Scientific Laboratory in the CMB Division under the supervision of Group Leader Morton C. Smith.

S U.S. GOVERNMENT PRINTING OFFICE: $1973-784.278 / 2$

\section{REFERENCES}

1. C. H. Dane and G. O. Backman, Cologic Map of New Mexico (U.S. Geological Survey, 1965).

2. D. W. Hyndman, Petrology of Igneous and Metamorpbic Rocks (McGraw-Hill, New York, 1972), p. 34.

3. Rock-Color Chart (Rock-Color Chart Committee, Geological Socicty of America, New York, 1963).

4. David B. Slemmons, "Determination of Volcanic and Plutonic Plagioclase Using a Three- or Four-Axis Universal Stage," Geological Society of America, Special Paper 69 (1962).

5. L. Van Der Plas and A. C. Tobi, "A Chart for Judging the Reliability of Point Counting Results," Am. I. Sci. 263, 87-90 (1965). 